(Aus der II. internen Abteilung des Krankenhauses der Stadt Wien [Vorstand: Primararzt. Dr. J. Zaffron].)

\title{
Unspezifische Immunität bei der chronischen Lungentuberkulose.
}

\author{
Von
}

Dr. Ferd. Mattausch,

1. Assistent der Abteilung.

(Eingegangen am 2. Oktober 1922.)

Die Immunitätswissenschaft hat jahrzehntelang allen FleiB und Arbeitseifer allzu einseitig auf die Erforschung der streng spezifischen Erscheinungen eingestellt, wie sie uns im Krankheitsverlaufe entgegentreten, und dabei die Wichtigkeit der unspezifischen Abwehrkräfte zu wenig berücksichtigt. Und doch ist der Begriff der unabgestimmten Immunität so naheliegend. Denn wie oft werden Erregerangriffe abgewehrt, ohne daß es überhaupt zur Krankheit kommt. Dabei können spezifische Kräfte nicht mitgewirkt haben, denn sie bilden sich erst in dem schon durch Krankheit umgestimmten Körper. Wir stehen hier vor Abwehrkräften, die bereits normalerweise im Körper vorhanden sind und die sich unabgestimmt gegen die verschiedensten artfremden Stoffe abbauend richten können.

Der exste, der dem spätervon $M u c h$ geprägten Begriffe der unspezifischen Immunität nahegekommen ist, war Weichardt. Seine Theorie der Protoplasmaaktivierung $\left.{ }^{1}\right)^{2}$ ), d. h. der unspezifischen Leistungssteigerung verschiedenster Zellgebiete (, omnicell l6läre Leistungssteigerung "), aus zahlreichen mühevollenVersuchen entsprungen, weist nachdrücklich auf die normalerweise in den Körperzellen ruhenden Kräfte und ihre Steigerungsmöglichkeit hin. Mit dieserallgemeinen Leistungsfähigkeit der Zellen fällt aber keineswegs die Fähigkeit des Körpers zur Bildung unabgestimmter Abwehrkräfte gegen schädliche Reize aller Art zusammen. Denn die Abwehrbereitschaft des Organismus, die Fähigkeit, selbstrcgulierend gegen jede Störung des biologischen Gleichgewichtes Abwehrmaßnahmen zu treffen, mit einem Worte die Immunität kann zu- oder abnehmen, ohne daß3 es dabei zu einer Stärkung oder Schwächung der allgemeinen Leistungsfähigkeitaller Zellen im Sinne Weichardts gekommen ist. Umgekehrtkann diese gesteigert oder gesch wächt werden, ohne daßderImmunitätszustand des betreffenden Individuums dabei eine Besserung oder Verschlechterung erfährt.

Der Begriff der unabgestimmten Immunität deckt sich sonach mit dem Begriffe der Protoplasmaaktivierung nicht, sondern ist unabhïngig von den Weichardt schen Arbeiten von $\left.M u c h^{3}\right)^{4}{ }^{5}$ ) aufgestellt worden.

Den Anstoß dazu gaben die Forschungen Fraenkels und $\left.M u c h s^{6}\right)$, denen es 1911 gelungen war, Meerschweinchen gegen einen äußerst giftigen Stamm von Paratyphus B (Gallebacillus Fraenkel-Much), der in jedem Falle bei diesen Tieren eine eitrige Gallenblasenentzündung hervorruft und zum Tode führt, durch Behandlung mit normaler Meerschweinchengalle völlig zu schützen. Diese Immunisierung wurde also nicht durch abgestimmte, sondern durch unabgestimmte Stoffe herbeigeführt?). Später gelang dann $A d a m^{8}$ ) durch einen dem Bacillus 
mesentericus nahestehenden harmlosen Luftkeim der Schutz von Meersch weinchen gegen Typhus- und Gallebazillen und auch Much und Schmidt ${ }^{9}$ ) konnten Tiere gegen diese höchst giftigen Erreger durch Impfung mit ungiftigen Luftspaltpilzen, Schimmelpilzen, Menschenfett und Menschengalle immunisieren.

$\left.M u c h^{10}\right)$ grenzte dann den Geltungsbereich der beiden Immunitätsarten gegeneinander ab und wies der spezifischen Imm unität ihre Hauptaufgabe bei den ganz chronischen Infektionskrankheiten, der unspezifischen Immunität bei den meisten akuten Erregererkrankungen zu, während die subakuten beider Immunitätsarten bedürfen.

Die praktischen Folgerungen aus ihren Forschungen haben beide Schulen bald gezogen: Aus der Weichardtschen Lehre der Protoplasmaaktivierung entstand die Proteinkörpertherapie, von $R$. Schmidt $\left.{ }^{11}\right)^{12}$ ) praktisch ausgebaut, zusammenfassend dargestellt von Kaznelson ${ }^{13}$ ), die aber leider nur dem einseitigen Dogma, daß Eiweiß allein die nötigen Immunkörper erzeugen könne, gerecht wird und die wichtige Rolle der Lipoide und Fette in der pathologischen Biologie nicht berücksichtigt; und Much selbst gab seinen unabgestimmten Impfstoff ,Immunvollvakzine“" heraus, der den drei großen reaktiven Gruppen aller Erregerstoffe (Eiweiß, Lipoid und Fett) Rechnung trägt und bei einer schweren Grippeepidemie die ersten guten Dienste leistete ${ }^{14}$ ).

Die Lungenphthise nun als eminent chronische Erkrankung fällt nach Much in den Geltungsbereich der spezifischen Immunität. Infolge der durch Monate und Jahre dauernden Erregerreize sind die Körperzellen bereits trainiert, gegen die stets gleichartigen Antigene spezifisch eingestellte Abwehrkräfte zu produzieren. Man wird daher bei Tuberkulösen, soweit sie ïberhaupt noch einer Reiztherapie zugänglich sind, in erster Linie bestrebt sein, mit spezifischen Vakzinen, also z. B. mit den Partigenen, mit denen die abgestimmte Behandlung durch Zerlegung in ihre einzelnen Reizfaktoren gewisscrmaßen auf die Spitze getrieben wird, oder auch mit anderen Tuberkelbazillenpräparaten das bereits funktionierende spezifische Abwehrsystem zu noch stärkerer Tätigkeit anzuregen.

Was aber mit jenen Fällen, bei denen es infolge anhaltender übermächtiger Erregerangriffe zur Überreizung, zur Zellähmung gekommen ist, zu jenem vollständigen Niederbruch des Durchseuchungswiderstandes, den Hayek ${ }^{15}$ ) negative Anergie nennt? Die total erschöpften Körperzellen reagieren hier auf keinen starken oder schwachen spezifischen Reiz mehr im Sinne einer Anregung der Abwehrtätigkeit, sondern nur mehr im Sinne der Lähmung. Hier laann, wenn überhaupt noch etwas, nur die unspezifische Immunität helfen. Diese wird man natürlich in erster Linie mit allen möglichen von altersher bekannten hygienischen und diätetitischen Maßnahmen zu heben trachten, evtl. aber auch mit einer unspezifischen Reiztherapie im engeren Sinne, mit anspezifischen Vakzinen. Nur darf man dabei nicht vergessen, daß im tuberkulös umgestimmten Körper infolge der wichtigen Rolle, welohe die Fett- und Lipoidstoffe bei der spezifischen Tuberkuloseimmunität spielen, auch die unabgestimmten Antilipoide und Antifette, die Lipasen im Sinne Bergels ${ }^{16}$ ), in den Vordergrund treten.

Vielleicht ist die seit altersher bekannte günstige Wirkung fetthaltiger Nahrungsmittel für den Verlauf der Tuberkulose auf das Auftreten unabgestimmter gegen die Fette gerichteter Abwehrfermente im Blute zurückzuführen - konnten doch Abderhalden und Rona ${ }^{17}$ ) auf enterale Zufuhr von Fetten eine vermehrte 
lipolytische Kraft des Serums feststellen; vielleicht gehört auch die Leberthranwirkung hierher, sicher wirkt aber das Chaulmoograöl bei Lepra und Stöltzners Tebelon ${ }^{18}$ ), der Isobutylester der Ölsäure, in erster Linie durch Stärkung der unspezifischen Lipoid- und Fettantikörper.

Ich selbst konnte mit dem von Markovic, Raskin und Jentzer angegebenen Versuchspräp. $671 \mathrm{E}$, einem Gemisch komplizierter gebauter Fett- und Lipoidstoffe, welche den gleichen Gruppen des Tuberkélbacillus chemisch nahestehen, bei einer Anzahl schwerer, zum Teil sogar prognostisch ungünstig zu beurteilender Phthisiker recht gute Erfolge erzielen ${ }^{19}$ ).

Wie können nun die unspezifischen Fettstoffe hier überhaupt wirken? Die spezifisch auf die $A b w e h r$ der Tuberkelbazillenantigene eingestellten Körperzellen sind bei den schweren progredienten Lungenphthisen durch den andauernden Krankheitsreiz bereits abgestumpft, gelähmt; eine spezifische Vakzine, ein Reiz also, der wieder auf dieselbe Art die bereits gelähmten Zellen trifft, kann nichts mehr nützen, höchstens schaden. Ein anderer Reiz aber, ein unabgestimmter, kann, wie Much ausführt, die Lähmung der gleichen Zellen, wenn sie noch keine vollständige war und sich nur auf den bestimmten spezifischen Reiz bezogen hatte, wieder beheben. Es wird dabei zunächst zur Bildung unspezifischer, später aber selbst wieder spezifischer Abwehrkräfte kommen.

Die Umbildung unabgestimmter zu abgestimmten Antikörpern ist übrigens eine in der pathologischen Biologie bereits bekannte Tatsache: Bergel $\left.\left.{ }^{18}\right), M u c h^{5}\right)$, auch Weichhardt und Schrader ${ }^{2}$ ) haben darauf hingewiesen. H. Schmidt ${ }^{20}$ ) sagt, da B nach unseren gegenwärtigen Kenntnissen zwischen den unabgestimmten Fermenten des Körpers und den hochgradig abgestimmten Antikörpern nur ein gradueller Unterschied besteht.

Zur spezifischen Einstellung der Reizantwort, zur Bildung abgestimmter Antikörper, kommt es zweifellos erst im Laufe einer dauernden Reizwirkung; das zum erstenmal an die Körperzellen kommende Erregerantigen kann ja zunächst nur unabgestimmt wirken, und erst die Umstimmung des infizierten Körpers macht Reiz und Reizantwort, Antigen und Antikörper zu spezifischen.

Wahrscheinlich geht die Umbildung unabgestimmter in abgestimmte $\mathrm{Ab}$ wehrkräfte um so leichter vor sich, je näher in ihrer chemischen Struktur sich das künstlich zugeführte Antigen und der krankmachende Erreger stehen. Die gün. stige Wirkung des oben angeführten Versuchspräp. $671 \mathrm{E}$ ist vielleicht in der nahen Verwandtschaft der darin enthaltenen Lipoid- und Fettstoffe mit den Tuberkelbazillenlipoiden und -fetten begründet.

Eine bedeutsame Stellung muß der unspezifischen Immunität im Verlaufe der chronischen Lungentuberkulose auch dann zukommen, wenn es zur akuten Proliferation eines früher chronischen Prozesses oder zu einer starken Herdreizung bei einer spezifischen Behandlung gekommen ist. Dem plötzlichen Überhandnehmen der Antigenwirkung sind die abgestimmten Abwehrkräfte hier nicht mehr gewachsen, sonst wäre es ja nicht zur Verschlechterung gekommen. Vielleicht kann man durch Anregung unabgestimmter Antikörper in diesem Zeitpunkte Besserung erwirken. Untersuchungen darüber, teils mit Immunvollvakzine, teils mit Versuchspräp. $671 \mathrm{E}$, teils mit anderen unspezifischen Mitteln sind im Gange, aber noch nicht abgeschlossen, so daß später erst zusammenhängend darüber berichtet werden kann. 
Weiters spielt die unabgestimmte Immunität eine wichtige Rolle bei jenen mit hohem intermittierenden Fieber einhergehenden tuberkulösen Erkrankungen, wo die starken Temperaturschwankungen durch Mischinfektionen bedingt sind. Hier können unspezifische Abwehrkräfte allein helfen.

Ich kann über 4 Fälle berichten, wo es mir gelungen ist, bei interkurrent im Verlaufe chronischer Tuberkulose aufgetretenen Mischinfektionen, klinisch durch bronchopneumonische Erscheinungen gekennzeichnet, durch unabgestimmte Reizbehandlung, nämlich durch Einspritzungen von Immunvollvakzine (Omnadin) nach $M u c h^{*}$ ) Entfieberung; Aufhören der schweren toxischen Erscheinungen und Schwinden der pneumonischen Infiltrationsherde zu erreichen.

Die 4 Beispiele seien hier angeführt.

1. J. $J$., 27 Jahre, Schlosser. Im Krieg 1918 als Matrose lungenkrank geworden. Seit 1 Monat starke Verschlechterung des Zustandes. Aufnahmebefund 9. II. 1921 : Progredients offene kavernöse Phthise des rechten Oberlappens, cirrhotisch-nodöse Tuberkulose der übrigen rechten Lunge mit begleitender Bronchitis des linken Oberlappens, geringe Exsudatreste rechts.

LobuläreHerde imlinken Unterlappen. Temperaturintermittierend febril(bis $39,4^{\circ}$ steigend).

Dyspnöe, leichte Cyanose. Puls 148, celer.

Sputum: Tuberkelbazillen positiv, der gewaschene Sputumkern enthält reichlich grampositive Kokken (Strepto- und Staphylokokken). Da die Diagnose auf eine im Verlauf einer chronischen Lungenphthise aufgetretene Kokkenmischinfektion gestellt wird, die im linken Unterlappen $\mathrm{zu}$ lobulärpneumonischen Herden geführt hat, wurden Versuche mit Immunvollvakzine (I. V. V.) gemacht.

12. II. $10^{\mathrm{h}}$ a. m. $: 2 \mathrm{ccm}$ I. V. V. intramuskulär $+4 \mathrm{cem}$ Ol. camphor. Temperatur bis $38,5^{\circ}$.

13. II. Temperatur bis $39,0^{\circ} 4^{\mathrm{h}}$ p. m.: 2 com I. V. V.

14. II. Temperatur afebril. Rasseln über dem linken Unterlappen gesehwunden, über der rechten Lunge namentlich die bronchitischen Geräusche viel spärlicher.

15. II. Afebril. Cyanose und Dyspnöe geschwunden.

16. II. Höchste Temperatur $37,1^{\circ}$. Rasseln rechts wieder etwas reichlicher, $4^{\mathrm{h}}$ p. m.: $2 \mathrm{ccm}$ I. V. V.

17. II. Höchste Temperatur $37,2^{\circ}$.

18. II. Afebril. Deutliches Nachlassen aller subjektiven Beschwerden und Verminderung der Rasselgeräusche überall. Lobuläre Herde über dem linken Unterlappen nicht mehr nachweisbar. Puls 92, ziemlich gut gespannt. 6 Wochen später kam es dann zuerst zu einer ausgedehnten exsudativen Pleuritis rechts, die zwar nach Punktion zurückging (spezifische Behandlung wurde nie vertragen), aber schließlich trat eine tuberkulöse Aussaat in Lunge, Kehlkopf und Darm auf (Exitus letalis 12. VII. 1921).

2. L. L., 45 Jahre, Agent. Lungenkrank seit 14 Jahren, schlechter seit 2 Jahren.

Aufnahmebefund 23. X. 1920: leicht progrediente offene kavernöse Phthise des linken Oberlappens, cirrhotisch-nodöse Tuberkulose des rechten Oberlappens und der Unterlappenspitze. Temperatur remittierend subfebril. Ab 2. XII. Bazillenemulsion in langsam steigenden Dosen, wegen Herzbeschwerden Kur abgebrochen.

12. III. 1921 plötzlich heftiger Schüttelfrost, Temperaturanstieg bis $39,8^{\circ}$, Stechen über der linken Lunge. Kleine bronchopneumonische Herde im linken Unterlappen wahrscheinlich. $4^{\text {h }}$ p. m.: 2 ccm I. V. V. intramuskulär. 13. III. Temperatur bis $40,0^{\circ}$. Dichtes Giemen und Pfeifen über der ganzen Lunge. Über dem linken Unterlappen stellenweise broncho. vesikuläres Atmen und feinblasiges konsonierendes Rasseln. $12^{\mathrm{h}} \mathrm{m.:} 2 \mathrm{ccm}$ I. V. V.

14. III. Temperatur bis $39,9^{\circ}$. Im gewaschenen Sputumkern massenhaft grampositive Kokken neben Tuberkelbazillen.

15. III. Temperatur bis $39,8^{\circ} .1 / 2^{1} 1^{\mathrm{h}}$ a. m.: $2 \mathrm{~cm} \mathrm{I.} \mathrm{V.} \mathrm{V:}$

16. III. Temperatur mittags nur mehr bis $38,2^{\circ}$ angestiegen, dann Abfall auf $36,9^{\circ}$.

Ab 17. III. Temperatur afebril. Giemen und Pfeifen über der ganzen Lunge deutlich nachgelassen, bronchovesikuläres Atmen links hinten unten geschwunden, nur mehr spärlich feinblasiges, nicht konsonierendes Rasseln.

*) Hergestellt durch die Firma Kalle \& Co., Biebrich a. Rh. 
Pat. wird am 14. IV. 1921 wesentlich gebessert entlassen, seine Lungenphthise hat deutlich den Charakter einer schrumpfenden, zur Indurierung neigenden angenommen, was durch eine Nachuntersuchung nach ca. I Jahre bestätigt gefunden wird.

3. J. K., 22 Jahre, Beamter. Seit 4 Jahren lungenkrank. Aufnahmebefund vom 28. XII, 1920: leicht progrediente cirrhotisch-nodöse Phthise beider Oberlappen und Unterlappenspitzen sowie der linken Hilusgegend. Auch hier kam es auf dem Boden der chronischen Tuberkulose am 5. II. 1921 nach Schüttelfrost und Temperaturanstieg auf $39,9^{\circ} \mathrm{zu}$ bronchopneumonischen Herden im rechten Unterlappen. Innerhalb von 5 Tagen 3 mal je $2 \mathrm{ccm}$ I. V.V. intramuskulär. Ab 10. II. blieb Patient nach fast kritischem Temperaturabfall anhaltend fieberfrei, die bronchopneumonischen Verdichtungserscheinungen schwanden wieder.

Später wurde Pat. mit Bazillenemulsion behandelt und am 1. VII. 1921 wesentlich gebessert (Lungenbefund zeigt deutliche Neigung zur Indurierung und Schrumpfung) entlassen. Die Nachuntersuchung nach $1 / 2$ Jahr ergibt eine cirrhotische stationäre Phthise.

4. $A$. C., 29 Jahre, Schlossergehilfensgattin. Iungenkrank seit 3 Jahren. Spitalsaufnahme 5. IV. 1922. Stationäre cirrhotisch-nodöse Tuberkulose beider Oberlappen und Hilusgegenden.

Am 22. VI. kam es nach Schüttelfrost zum Temperaturanstieg auf $40,3^{\circ}$ und Ausbildung bronchopneumonischer Herde im linken Unterlappen, die dann auf die Pleura übergriffen und schließlich per continuitatem eine Pericarditis externa erzeugten. Temperatursteigerungen über $39,0^{\circ}$ wurden jedesmal durch I. V. V.-Injektion prompt herabgedrückt und waren dann von einer fieberfreien Periode gefolgt. Das wiederholte sich 3 mal, jedesmal gelang es durch $2 \mathrm{ccm} I$. V. V. die neuerlich aufgeflackerte Mischinfektion wieder zur Ruhe zu bringen.

Alle 4 Fälle zeigen eine auf dem Boden einer schweren Phthise interkurrent aufgetretene Bronchopneumonie, hervorgerufen durch Kokkenmischinfektion. Trotz der großen Gefahr, die darin lag, daß der zweite Infekt die Abwehrkraft des Körpers zu sehr in Anspruch nehmen und es dadurch zu einer akuten Proliferation der Phthise kommen könnte, klingt die Bronchopneumonie nach einigen I. V. V.Injektionen rasch ab. Bei Fall 1 kommt es allerdings - aber erst nach Wochen nach Ausbildung einer exsudativen tuberkulösen Pleuritis zum Niederbruch des Durchseuchungswiderstandes. Fall 2 und 3 erleben aber eine langsam fortschreitende und anhaltende Besserung ihrer chronischen Tuberkulose. Vielleicht wurden gerade durch die Einverleibung der unspezifischen Vakzine nicht nur die unabgestimmten, sondern auch die abgestimmten Abwchrkräfte angeregt, so daß die nachfolgende spezifische Vakzinebehandlung (Bazillenemulsion) bei Fall $3 \mathrm{um}$ so mehr Wirksamkeit entfalten konnte. Damit wäre ja eigentlich die Muchsche Forderung ${ }^{14}$ ) erfüllt, beide Immunitätsarten auszunützen und eine unabgestimmte Vollvakzine als Aktivator jeder abgestimmten Immunität zu benützen, auf die man dann die abgestimmte Vakzine aufpflanzen kann.

Bei Fall 4 ist wohl nach jeder I. V. V.-Injektion mit der Wiederholung derselben zu lange gewartet worden, so daß die Erreger der Mischinfektion immer wieder Kräfte zu neuem Angriff sammeln konnten, sonst hätten sich die Temperatursteigerungen nach den Erfahrungen bei don ersten 3 Fällen nicht noch 2 mal wiederholt.

Daß es nach Einverleibung von I. V. V. zu einer bedeutenden Steigerung des Lipasegehaltes im Blutserum, also zu einer Zunahme unabgestimmter Abwehrkräfte wenigstens gegen Fett- und Lipoidantigene kommen kann, ist als zufäliger Nebenbefund in einem anderen Falle erhoben worden.

5. $R$. K., 25 Jahre, Lehrer. Seit 3 Jahren lungenkrank. Progrediente nodöse Phthise beider Ober- und des linken Unterlappens, im linken Oberlappen nußgroße Kaverne. Pat. erhielt mit gutem Erfolge eine kombinierte Behandlung mit Präp. $671 \mathrm{E}$ und Tuberkulin. Im Februar 1922 trat eine interkurrente grippeartige Erkrankung auf: mehrtägiges Fieber bis $39^{\circ}$ mit starken allgemeinen toxischen Beschwerden, Laryngitis, Tracheobronchitis. Nach 3 mal $2 \mathrm{ccm}$ I. V. V. intramuskulär war der Prozeß innerhalb von 5 Tagen abgeklungen. 
Bei dem Pat. wurde der Wert des Serumlipasetiters fortlaufend allmonatlich nach der von Bach angegebenen, von Raskin modifizierten Methode der Guajakolkarbonat-Aufspaltung gemessen. Der im September 1921 entsprecher,d der ziemlich ungünstigen Prognose recht niedrige Lipasewert des Blutserums von 0,023 stieg im Oktober 1921 auf 0,031 und blieb bis Februar 1922 mit klcinen Schwankungen zwischen 0,030 und 0,033. Die Messung nach der 3. I. V. V.-Injektion ergab den Wert von 0,049, also eine besondere Steigerung der aufspaltenden Kraft des Serums nach der Einverleibung der unabgestimmten Vakzine. Die Zunahme des Lipasetiters ist um so auffallender, als man ja kurz nach Abklingen der grippeartigen Erkrankung infolge Aufbrauchung aller Schutzkräfte eher eine starke Abnahme aller Reaktivität erwartet hätte. (Die angeführten Zahlen sind nicht absolute Wertangaben etwa für die im Kubikzentimeter Blutserum enthaltene Lipasemenge, sondern haben lediglich die Bedeutung von relativen Werten. Das soll z. B. heißen, daß eine bestimmte Serummenge im September 0,023 ccm Guajakolkarbonat aufzuspalten imstande war, während die gleiche Serummenge im Oktober 0,031 ccm zerlegen konnte, was natürlich einer höheren Spaltungskraft entspricht.)

Neben der von Schmidt und Peemöller ${ }^{14}$ ) angegebenen guten Wirksamkeit der Imunvollvakzine bei Grippe und Grippepneumonie und der von Send ${ }^{21}$ ) beobachteten Erfolge bei Puerperalfieber, septischem Abortus, Erysipel und Sepsis kämen als weiteres wichtiges Indikationsgebiet zur Anwendung des Omnadins sonach noch die im Verlaufe einer chronischen Lungenphthise interkurrent auftretenden bronchopneumonischen Mischinfektionen in Betracht. Wieweit die Vakzine zur Hebung der unabgestimmten Abwehrkräfte auch bei der unkomplizierten Tuberkulose sonst noch brauchbar ist, werden weitere Versuche lehren.

Ebenso bleibt es einer späteren Veröffentlichung vorbehalten, Untersuchungen zu besprechen, welche die Steigerung der unabgestimmten Immunität durch verschiedene unspezifische Heilmaßnahmen und deren Einfluß auch auf die abgestimmten Abwehrkräfte maßanalytisch klarlegen sollen.

Zweck dieser Zeilen ist es vor allem, zu zeigen, wie die $M u c h$ schen Forschungen über das Problem der unabgestimmten Immunität, deren hauptsächlichster Wirkungskreis ohne $Z$ weifel die akuten und subakuten Krankheiten sind, auch bei der so eminent chronischen Lungenphthise fruchtbringende Verwertung finden können.

\section{Literaturverzeichnis.}

$\left.{ }^{1}\right)$ Weichardt, Münch. med. Wochenschr. 1918, Nr. 22. - ${ }^{2}$ ) Weichardt und Schrader, ebenda 1919, Nr. 11. - 3) Much, Pathologische Biologie (Immunitätswissenschaft). Lehrbuch. 3. Aufl. Kabitzsch, Leipzig. $\left.-{ }^{4}\right)$ Much, Die Partigengesetze und ihre Allgemeingültigkeit. Kabitzsch, Leipzig 1921. $\left.-{ }^{5}\right)$ Moderne Biologie, Heft 1: Much, ther die unspezifische Immunität. Heft 2 und 3: Much, Spezifische und unspezifische Reiztherapie. Kabitzsch, Leipzig 1921 und 1922. $-{ }^{6}$ ) Fraenkel und Much, Zeitschr. f. Hyg. u. Infektionskrankh. 69. 1911. - 7) Much, Dtsch. med. Wochenschr. 1919, Nr. 26. - 8) Adam, Beitr z. Klin. d. Infektionskrankh. u. z. Immunitätsforsch. 3, H. 1/2. 1914. (Eppendorfer Festschrift.) $\left.{ }^{9}\right) \mathrm{Much}$, Dtsch. med. Wochenschr. 1920, Nr. 18. - $\left.{ }^{10}\right)$ Much, ebenda 1920, Nr. 29. 11) Schmidt, $R$. und Kraus, Med. Klinik 1919, Nr. 22. - ${ }^{12}$ ) Schmidt, $R$. und Kraus, ebenda 1920, Nr. 27. - ${ }^{13}$ ) Kaznelson, Die Grundlagen der Proteinkörpertherapie. In Weichardts Ergebn. d. Hyg., Bakteriol., Immunitäts-Forsch. u. exp. Therapie 4. Springer, Berlin 1920. 14) Much, Schmidt und Peemöller, Müneh. med. Wochenschr. 1920, Nr. 37. - 15) Hayek, Das Tuberkuloseproblem. Springer, Berlin 1920. - ${ }^{16}$ ) Bergel, Die Lymphocytose, ihre experimentelle Begründung und biologisch-klinische Bedeutung. Springer, Berlin 1921. 17) Abderhalden und Rona, Zeitschr. f. phys. Chemie 75. 1911. - 18) Stöltzner, Münch. med. Wochenschr. 1919, Nr. 24 und 1920, S. 1196. - 19) Publikation im Druck. - ${ }^{20}$ ) Moderne Biologie, Heft 4 und 5: Schmidt, H., Zur Biologie der Lipoide. Kabitzsch, Leipzig 1922. 21) Send, Dtsch. med. Wochenschr. 1922, Nr. 33. 